\title{
Clinical Study: Lotion Containing Propolis Special Extract GH 2002 0.5\% vs. Placebo as On-Top Treatment of Herpes Zoster
}

\author{
Denisa Tomanova ${ }^{1}$, Simona Holcova ${ }^{1}$, M. Hladíková ${ }^{*}$ \\ ${ }^{1}$ Dermatological Ambulatorium, Consultancy of Dermatovenerology, Brno, Czech Republic \\ ${ }^{2}$ Department of Medical Informatics, 2nd Faculty of Medicine, Charles University of Prague, Prague, Czech Republic \\ Email: simona.holcova@email.cz, *statistika.hladikova@seznam.cz
}

How to cite this paper: Tomanova, D., Holcova, S. and Hladíková, M. (2017) Clinical Study: Lotion Containing Propolis Special Extract GH 2002 0.5\% vs. Placebo as On-Top Treatment of Herpes Zoster. Health, 9, 1337-1347.

https://doi.org/10.4236/health.2017.910097

Received: May 23, 2017

Accepted: September 19, 2017

Published: September 22, 2017

Copyright (๑) 2017 by authors and Scientific Research Publishing Inc. This work is licensed under the Creative Commons Attribution International License (CC BY 4.0).

http://creativecommons.org/licenses/by/4.0/

\begin{abstract}
Background: The aim of the clinical trial was to establish the contribution of propolis special extract GH 2002 as constituent of a lotion for the supportive treatment of Herpes zoster. Methods: 60 patients with diagnosed Herpes zoster were either treated with a propolis containing $(n=33)$ or an identical, but propolis-free lotion $(\mathrm{n}=27)$ for 28 days, as an add-on therapy to oral antiviral treatment with aciclovir. Study parameters were the assessment of pain on a visual analogue scale, the assessment of the formation of new vesicles, and the safety of application of the preparation. Results: Improvement of pain was significantly better and quicker in the propolis lotion group $(\mathrm{p}<0.001$ at all visits). On day $3 / 4$, the reduction of pain versus baseline was $54 \%$ versus $20 \%$ reduction in favour of propolis lotion. On day 14 the results were $80 \%$ versus $42 \%$ pain reduction. Healing of lesions was significantly quicker with the propolis-containing lotion: at visit 3 on day 7, 21\% versus none of the patients showed a closed skin with or without crusting. After 14 days, the comparison showed $57.6 \%$ versus $25.9 \%$. At least $50 \%$ of propolis-treated patients were lesion-free on day 14 , versus day 28 in the control group $(\mathrm{p}=0.013)$. The formation of new vesicles was also significantly suppressed. On day $3 / 4$ the number of newly formed vesicles was 15 versus 25 , on day seven the number of new vesicles was 4 versus $14(\mathrm{p}<0.001)$. The advantage of propolis lotion was confirmed through the global assessment of efficacy by the physician, with 75.8 versus $33.3 \%$ "good" to "excellent" ratings already on day $3 / 4,90.9$ vs. $44.4 \%$ on day 7 , and 100 vs. $74.1 \%$ on day 28 (p $<0.001$ ). Skin tolerability was excellent, no allergic reactions, skin irritations or other adverse events were observed. Conclusion: The observations clinically confirm antiviral and anti-inflammatory effects of propolis special extract GH 2002 and at the same time demonstrate the benefits of the topical add-on to the systemic antiviral therapy of Herpes zoster.
\end{abstract}




\section{Keywords}

Herpes Zoster, Propolis, Controlled Clinical Trial

\section{Background}

The Varicella zoster virus-also the cause of chickenpox in childhood-causes a typical rash known as Herpes zoster. Inflamed vesicles are typically spread over limited skin areas, mostly on the upper body. The diagnosis is based on the inflamed skin areas, the vesicles and the characteristic location and form of the eczema [1]. Treatment recommendations supported by guidelines [2] [3] and based on clinical evidence [4] [5] are the oral administration of virostatics such as brivudin and aciclovir. A major concern during therapy is the skin inflammation with strong itching and pain [6] [7], which are not directly influenced by antiviral remedies. These symptoms are usually addressed through the additional application of topical remedies such as zinc lotions and/or locally anti-inflammatory, antiseptic and soothing preparations. Even the use of local anaesthetics has been evaluated [8]. Experience shows that in most cases the outcome of such on-top measures is frequently not satisfactory for the patient or hard to fit into the patient's daily routines.

A promising natural product with distinct antiviral and anti-inflammatory properties is propolis. In an earlier clinical single-arm observational study we evidenced antiviral effects with cutaneous application of a lotion with propolis special extract GH 2002 as an add-on to guideline-conforming antiviral oral therapy of Herpes zoster. Pain, itching and burning as the most burdensome symptoms of Herpes zoster were effectively improved or even eliminated by the regular application of the lotion [9].

The preparation "GH 2002" used for the manufacture of the lotion applied in this study is a purified and standardized extract of propolis obtained from controlled sources in a closely defined region in the Czech Republic. The extract contains a reproducible quantity of flavonoids, polyphenols and phenyl carboxylic acids. Manufacturing involves purification by removal of potential sensitising constituents. Walgraves et al. (2005) reported an incidence of $1.2 \%$ to $6.6 \%$ of patients revealed as sensitive to propolis in patch-testing with 3-methyl-2-butenyl caffeate and phenylethyl caffeate being the major constituents responsible for the effect [10]. These authors therefore recommend specific patch-testing for dermal preparation such as lip balm and lotions containing propolis. In the case of GH 2002, the material has in fact been submitted to a patch test (unpublished results), which confirmed the safety of the extract.

Its antiviral, antibacterial and antiphlogistic as well as locally anaesthetic properties make propolis special extract GH 2002 especially suitable for local application, as demonstrated in clinical studies on the treatment of Herpes labialis [11] [12]. 
The antiviral effects of this extract were previously demonstrated in pharmacological studies with an emphasis on Herpes simplex virus I and II [13] [14] [15], and specifically also Herpes zoster virus (Schnitzler P. and Fickentscher H., unpublished study report 2008). In addition, the same extract was also found to have antimicrobial properties [15].

In commercial preparations propolis is not the only potentially active constituent. The study preparation also contains vitamin E, panthenol, (-)- $\alpha$-bis- abolol, phenoxyethanol and evening primrose oil. The same lotion was also applied in our open, observational study [9], where positive results were found in the treatment of Herpes zoster. Whereas there was undoubtedly a benefit of the application of the lotion, the question arose, which part of the observed effect could be attributed to propolis extract as a lotion component. The study described herein was therefore designed to assess the contribution of propolis special extract GH 2002 in the lotion by comparing the effects of the test product with those of a reference lotion of the same composition, but without propolis special extract.

\section{Methods}

\subsection{Ethics, Consent and Permissions}

The study was designed as an open trial in a single study centre at a dermatological ambulatory with an intended number of 60 participants (Trial Registration: ISRCTN17781274). This number was derived from previous experience with the study preparation [9], but was not formally established through a case number calculation.

The study was planned and carried out in accordance with the criteria of Good Clinical Practice (GCP) and the ethical standards defined in the declaration of Helsinki. As this was a non-randomized study with a cosmetic preparation given on top of the treatment recommended by treatment guidelines, an approval of an Ethics Committee was not required. Consent to study participation was received from all participants. Initiation of the trial was on 24 February 2012. The final visit of the last participant took place on 14 January 2014.

The intention to treat (ITT) group was defined as the patients who had received the study preparation or the reference product, and had returned for at least one visit after the admission examination. Per protocol group (PP) included all patients who followed the protocol in a reasonable manner. The safety population comprised all patients exposed to study medication.

\subsection{Inclusion/Exclusion Criteria}

Patients of both genders at the age of 18 - 70 years with no known hypersensitivity against any of the lotion constituents could be included after the diagnosis of Herpes zoster was made. Conditions for the inclusion were an appearance of the first vesicles within the last 72 hours, the occurrence of new vesicles within the last 24 hours, and an affection of mainly one skin segment of at least $3 \mathrm{~cm}^{2}$. Pa- 
tients were excluded when the manifestations occurred at the ears or the eyes, or any branch of the Trigeminus nerve. They could also not be included when they had a known hypersensitivity against any constituent of the lotion, or if they had a history of ophthalmologic, neurological or auditory complications or visceral dissemination during this or a previous episode of Herpes zoster. Immunodeficiency, cancer or major systemic diseases were further exclusion criteria. The participants were instructed not to use other treatments against itching, pain and inflammation, including topical products.

\subsection{Study Preparation and Reference Product}

Patients with diagnosed Herpes zoster were prescribed an oral antiviral treatment with aciclovir in the form of tablets (Herpesin, Teva Pharmaceuticals, Prague). Patients with a first manifestation of a Herpes zoster episode were told to take $400 \mathrm{mg}$ five times daily, whereas patients with former episodes were to take $800 \mathrm{mg}$ five times daily. The tablets were dispensed during the first visit, with pill counts on all subsequent visits for the assessment of compliance. In addition to this basis treatment, patients were given the study lotion or a reference lotion: Both were manufactured by Gehrlicher Pharmazeutische Extrakte (Eurasburg/Oberbayern, Germany). Both lotions contained vitamin E, bisabolol, panthenol and evening primrose oils as care-providing active substances. Further components according to the INCI declaration were aqua, propylene glycol, Oenenthera biennis oil, sodium PCA, cetearyl alcohol, octyldodecanol, ceteareth 30, stearic acid, phenoxyethanol, carbomer, sodium hydroxide, citric acid. The study preparation, but not the reference product, contained in addition special propolis extract GH 2002 at a concentration of $0.5 \%$. The undistinguishable lotions were dispensed by the physician upon inclusion, to be self-administered by the patient $2-3$ times daily on the affected skin area.

\subsection{Examinations}

The location of the Herpes zoster manifestation was recorded at the inclusion visit (data not further analysed), together with details such as previous episodes, co-medications and concomitant diseases. The status of the infection was documented on day 0 (Visit 1), with follow-up examinations on day $3 / 4$ (Visit 2), day $7 \pm 1$ (Visit 3), day $14 \pm 2$ (Visit 4) and day $28 \pm 2$ (Visit 5). A pill count and an inspection of the dispensed tube were made on all visit days to assess patient compliance with the oral and topical treatment.

Pain was assessed by the patient on each visit, using a visual analogue scale (VAS; 0 - $100 \mathrm{~mm}$ ). The status of the episode was documented by the physician. The course of the therapy was monitored through the evaluation of the time until inflammations and the erosive phase are ended with an incrustation of the lesions or a closed skin. In addition, the appearance of new vesicles during the treatment period was documented. The physician rated the treatment effect on a 4 -step verbal rating scale (poor-moderate-good-excellent). Adverse events and 
local tolerance were documented at each visit.

\subsection{Statistics}

IBM SPSS Statistics version 21.0.0 was used as the statistical software. P-Values from statistical analyses do not possess confirmatory value.

\section{Results}

60 patients of both sexes (39 female, $65 \%$ and 21 male, 35\%) were examined and treated. Thirty-three patients received the propolis GH 2002 lotion and 27 patients the reference lotion. The slight difference in group occupation (see results) was not intentional, but was caused by the packaging of four bottles of study preparation or reference in one cardboard box upon shipping from the manufacturer to the study centre. There were no drop-outs: All 60 patients returned for the follow-up visits. The PP population was therefore identical to the ITT population; i.e., $\mathrm{n}=60$ patients in both the ITT and PP population. The age distribution is given in Table 1.

10 patients of the group receiving the propolis lotion $(30.3 \%)$ and five patients receiving the reference lotion (18.5\%) reported previous episodes of Herpes zoster. Due to the small figures a subgroup analysis of the results by former episodes was not made. There was no significant difference between groups for age or gender distribution and for the occurrence of previous episodes of Herpes zoster (Fisher's exact test, $\mathrm{p}=0.38$ ).

\subsection{Effect on Pain}

The results of pain measurements on the visual analogue scale (VAS) are summarized in Table 2 and Figure 1. There was a highly significant difference for pain reduction in favour of the lotion containing propolis $(\mathrm{p}<0.001$ for each visit; nonparametric Mann-Whitney tests with correction for multiple comparisons). As was to be expected, pain reduction was also found with the reference preparation, but a clear effect was only found after $14 \pm 2$ days. Pain calculated as percent of baseline values differed highly significantly between groups at all visits ( $\mathrm{p}<0.001$ for each visit; Figure 2 ). Already on day $3 / 4$, the reduction of pain versus baseline was $54 \%$ with propolis lotion versus $20 \%$ reduction with the reference lotion. On day $14 \pm 2$ the results were $80 \%$ versus $42 \%$ pain reduction. With Herpes zoster being a self-limiting condition, the difference between groups decreased thereafter, but was still significant until day $28 \pm 2$.

An analysis according to the formula $\Delta$ Pain $=0.5 \times($ Pain [V3] + Pain [V4] $)-$ Pain [V1]) allows the visualisation of the effects attributable to the active con-

Table 1. Age distribution of the patients in years.

\begin{tabular}{cccccc}
\hline & $\mathrm{n}$ & Mean & Std. deviation & Minimum & Maximum \\
\hline Propolis lotion & 33 & 50.1 & 16.7 & 21 & 78 \\
Reference lotion & 27 & 45.3 & 18.9 & 18 & 78 \\
\hline
\end{tabular}


Table 2. Pain in the course of the study, measured in mm VAS.

\begin{tabular}{ccccccccc}
\hline & & \multicolumn{7}{c}{$95 \%$ confidence } \\
& & \multicolumn{7}{c}{ interval for mean } \\
\cline { 3 - 8 } & & Mean & $\begin{array}{c}\text { Lower } \\
\text { limit }\end{array}$ & $\begin{array}{c}\text { Upper } \\
\text { limit }\end{array}$ & Median & SD & Min & Max \\
\hline Visit 1 & Propolis & 66.5 & 61.9 & 71.1 & 72 & 13.0 & 35 & 84 \\
(Day 0) & Reference & 57.1 & 50.8 & 63.4 & 59 & 15.9 & 27 & 81 \\
Visit 2 & Propolis & 29.9 & 24.7 & 35.1 & 28 & 14.6 & 8 & 67 \\
$($ Day 3/4) & Reference & 42.9 & 36.4 & 49.5 & 40 & 16.6 & 12 & 75 \\
Visit 3 & Propolis & 17.8 & 14.1 & 21.6 & 14 & 10.6 & 5 & 47 \\
$($ Day 7 \pm 1$)$ & Reference & 38.1 & 32.7 & 43.5 & 37 & 13.7 & 9 & 73 \\
Visit 4 & Propolis & 12.4 & 9.4 & 15.5 & 9 & 8.6 & 4 & 44 \\
$\begin{array}{c}\text { (Day } \\
14 \pm 2)\end{array}$ & Reference & 30.9 & 25.5 & 36.2 & 32 & 13.4 & 8 & 58 \\
Visit 5 & Propolis & 9.2 & 6.6 & 11.8 & 7 & 7.3 & 3 & 34 \\
$\begin{array}{c}\text { (Day } \\
28 \pm 2)\end{array}$ & Reference & 24.5 & 20.4 & 28.7 & 25 & 10.4 & 5 & 48 \\
\hline
\end{tabular}

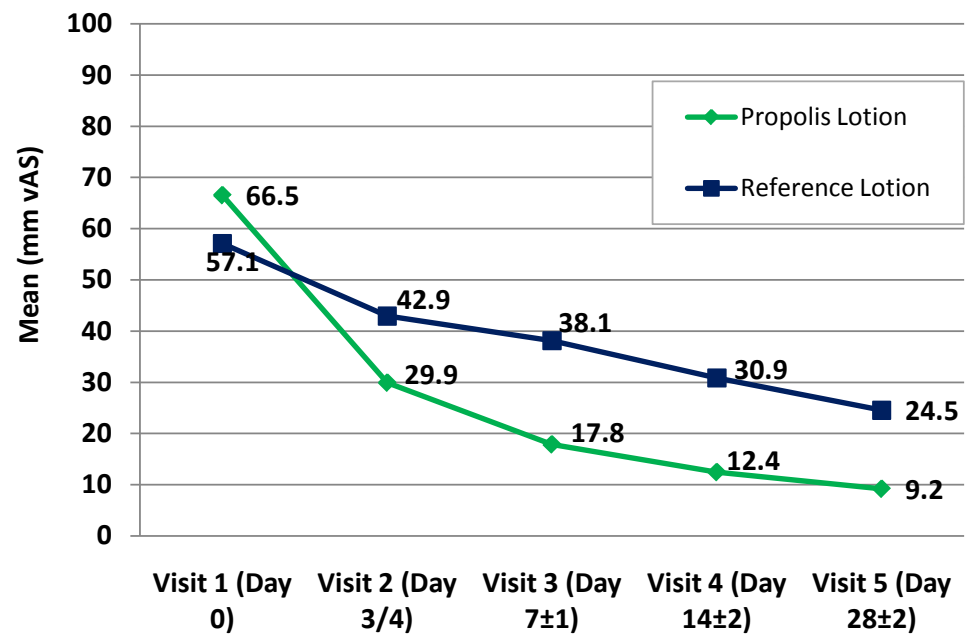

Figure 1. Reduction of pain during the study in absolute values.

stituent propolis special extract (Table 3, Figure 3). The difference was statistically significant ( $\mathrm{p}<0.0001$; Mann-Whitney-U-Test for independent samples).

\subsection{Stage of Herpes Zoster Lesions}

The cumulative number of patients with encrusted or closed lesions is displayed in Figure 4 for the various time points. The difference between groups represents the contribution of propolis extract against inflammation. It corroborates the results of the assessment of pain.

The statistical evaluation showed significant differences between the propolis lotion and the reference lotion. At visit 3 on day $7 \pm 1,21 \%$ of the patients applying propolis lotion versus none of the patients in the reference group showed 


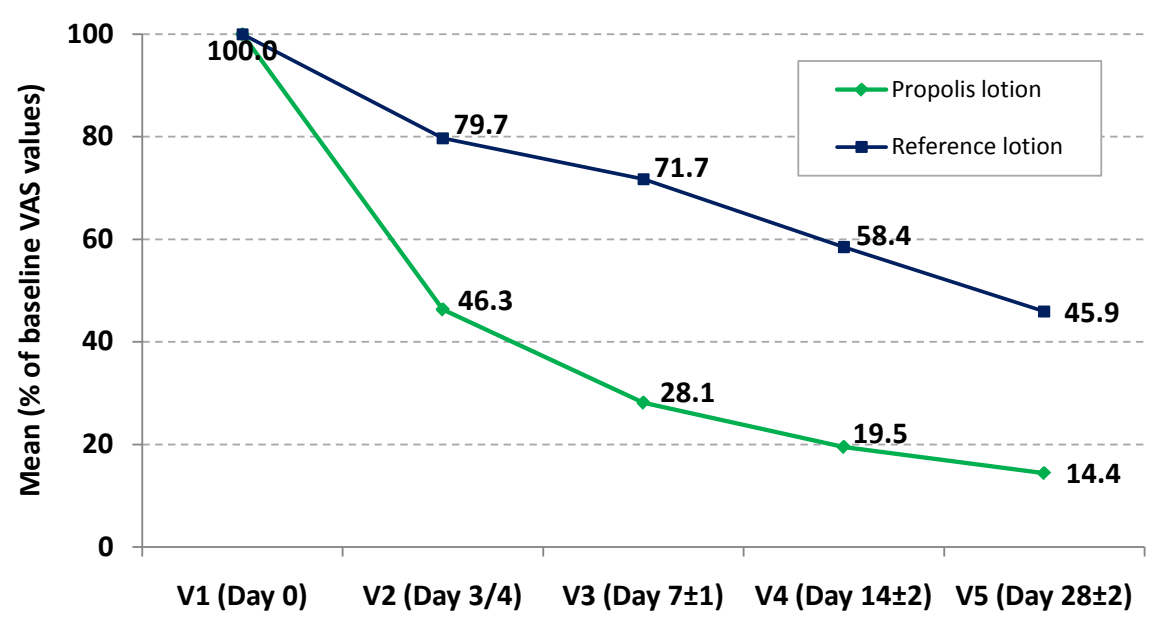

Figure 2. Pain reduction calculated as percent of starting value.

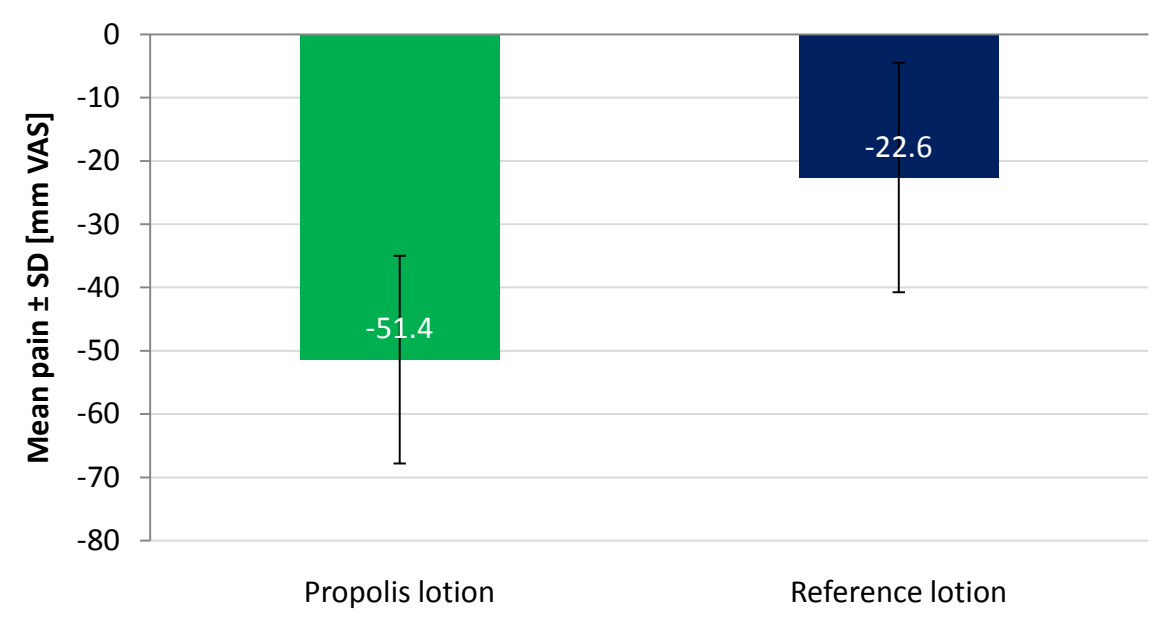

Figure 3. Difference of average pain at V3 and V4 to starting values at V1.

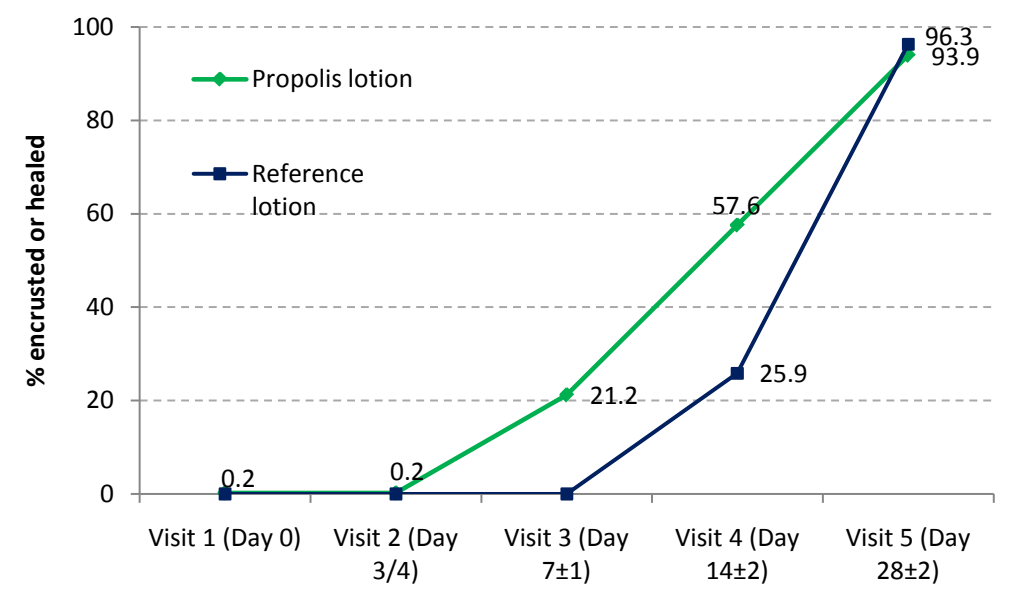

Figure 4. Cumulative percentage of crusted or healed Herpes zoster lesions in the course of the study.

a closed skin with or without crusting. After $14 \pm 2$ days, $57.6 \%$ of the propolis group and $25.9 \%$ of the reference group had reached this stage. After $28 \pm 2$ 
Table 3. Difference of average pain at V3 and V4 in mm VAS to starting values at V1. Negative values are improvements versus starting values.

\begin{tabular}{ccccccc}
\hline & $\mathrm{N}$ & Mean & Median & SD & Minimum & Maximum \\
\hline Propolis lotion & 33 & $-\mathbf{5 1 . 4}$ & -60.5 & 16.4 & -73 & -16.5 \\
Reference lotion & 27 & $-\mathbf{2 2 . 6}$ & -20.5 & 18.1 & -53 & 26.5 \\
\hline
\end{tabular}

days, there was no more difference between groups (94\% versus $96 \%$ ), which reflects the self-healing course of the episode. At this point there were only three patients left, which were not yet fully encrusted. Calculations of the time until at least $50 \%$ of study participants reached the stage of fully crusted or healed lesions indicated visit 4 at day $14 \pm 2$ for patients applying the propolis lotion, and visit 5 at day $28 \pm 2$ for those applying the reference lotion. The improvement in time to healing with propolis lotion was statistically significant (Mann-Whitney-Test, $\mathrm{p}=0.013$ ).

\subsection{Appearance of New Vesicles}

In the course of the study new vesicles appeared in both groups, but the number of newly formed vesicles was significantly lower in the verum group than with application of the reference lotion (Figure 5). On day $3 / 4$ the number of newly formed vesicles was 15 in the propolis group versus 25 with reference, on day $7 \pm$ 1 the difference was 4 versus 14 . At the following visits there was no more difference between groups ( 0 versus 1 new vesicle at day $14 \pm 2$, none thereafter). The difference between groups at visits 2 (day $3 / 4$ ) and visit 3 (day $7 \pm 1$ ) was statistically significant (Chi square test, $\mathrm{p}<0.001$ ).

\subsection{Overall Assessment by the Physician}

The global assessment of the course of the Herpes zoster development by the attending physician confirmed the results of the previous analyses (Figure 6). From visit 2 (day $3 / 4$ ) onwards, the group applying the propolis lotion received significantly better ratings than the reference group (Mann-Whitney test, $\mathrm{p}<$ $0.001)$.

\subsection{Local Tolerance/Adverse Events}

Both lotions were perfectly tolerated. No skin irritations, allergic reactions or other adverse effects were observed throughout the study.

\section{Discussion}

The patients of this study had a basic treatment with oral aciclovir during the entire duration of the individual episodes. Due to the presence of lotion constituents with known skin-care effects it may be expected that the propolis-free lotion also positively contributed to the overall effect, but the two contributions of oral aciclovir and the topical propolis-free lotion matrix cannot be singled out. The very same exposure to aciclovir and the lotion matrix was present in the 


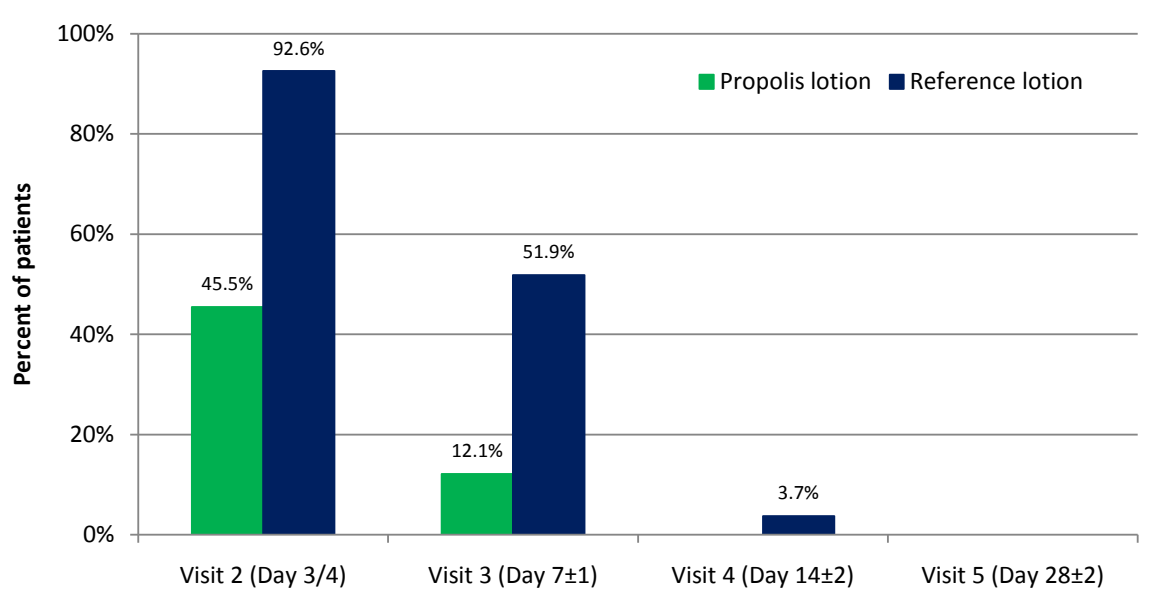

Figure 5. Formation of new vesicles in the course of the study.

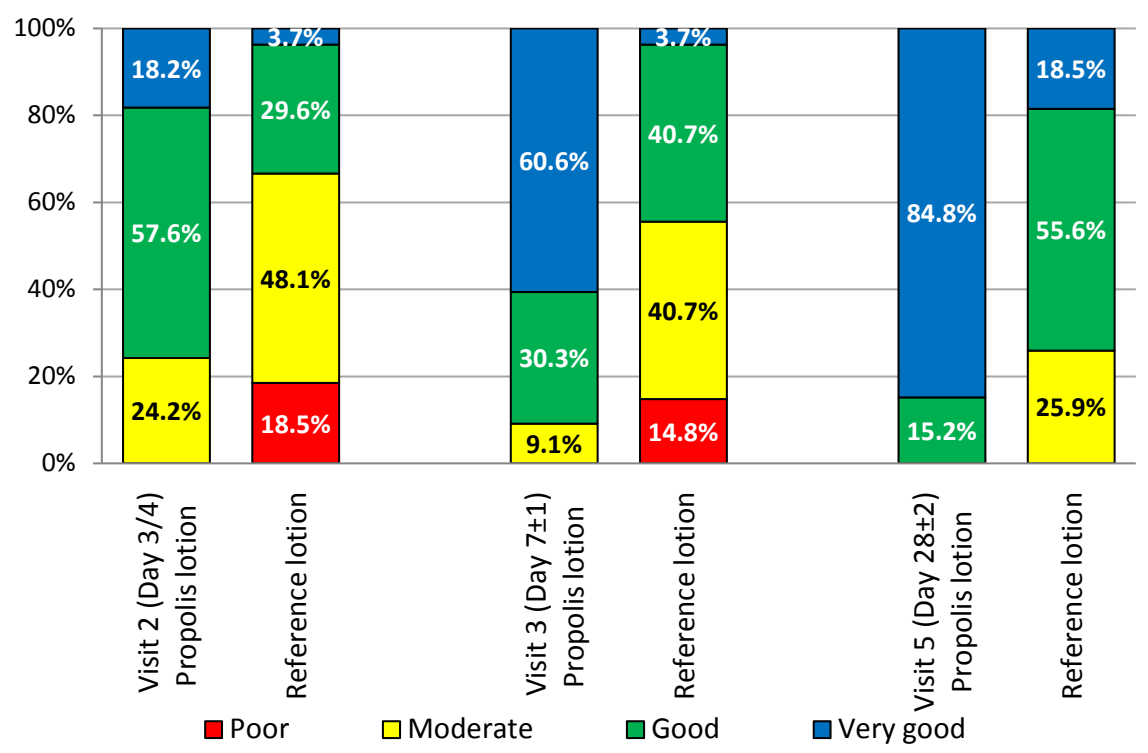

Figure 6. Global assessment of clinical effect by the physician.

propolis group, plus propolis extract. Any group differences are therefore associated with the additional application of the propolis extract. The higher dose of aciclovir in patients with a history of former episodes of Herpes zoster did not visibly impact the course of the episode in the corresponding patients, although no specific analysis was made due to the small case numbers.

The study results justify the addition of a topically applied remedy against local pain and itching to an oral antiviral treatment. According to our experience, the classical cutaneous application of zinc lotions or anti-inflammatory and antiseptic topical agents is not entirely satisfactory. Propolis special extract GH 2002, topically applied, had already shown positive effects against Herpes labialis [11] [12] and Herpes zoster [9] in earlier clinical investigations. The antiviral, anti-inflammatory and antimicrobial properties of this active constituent, evidenced in pharmacological examinations [13] [14] [15], most likely contributed to the distinct effects against pain and new vesicle formation observed in this 
study, especially in the early phases of the episode. The patient benefits from the quicker healing of skin erosions and less pain as compared to a propolis-free lotion, supported by an excellent tolerability.

Similar results were already obtained in our earlier study with the same lotion [9]: there was a distinct early pain relief measured by VAS (30\% reduction after 3 - 4 days, $\mathrm{p}<0.001$ ) and a full encrustation or healing of lesions in $55 \%$ of patients already after one week of application. The earlier results support the findings of this study.

\section{Conclusion}

The add-on therapy of pain and skin symptoms in episodes of Herpes zoster with a locally applied lotion containing propolis special extract GH 2002 as an active constituent adds distinct advantages to the oral antiviral therapy. With this study, the beneficial contribution of propolis extract as an antiviral and antiinflammatory active constituent of a topically applied lotion was evident: the comparison of an otherwise identical lotion with and without propolis allows attributing the beneficial differences between group to the active constituent $0.5 \%$ propolis special extract GH 2002 in the lotion. The topical application of a propolis containing lotion effectively supports the oral antiviral therapy by reducing pain and vesicle formation, and thus the most burdensome symptoms of a Herpes zoster episode.

\section{Competing Interests}

This clinical study was supported by funds from the company Gehrlicher Pharmazeutische Extrakte GmbH (Eurasburg, Germany; manufacturer of the study preparations). The sponsors were, however, not involved in the planning, execution, evaluation and publication of the clinical trial. These tasks were carried out under the responsibility of the authors.

SH and DL were the investigators, treating the patients and documenting the results. MH was responsible for the study plan and the statistical evaluation of the results.

\section{References}

[1] Kempf, W. and Lautenschläger, S. (2001) Infektionen mit dem Varizella Zoster Virus. Hautarzt, 52, 359-376. https://doi.org/10.1007/s001050051326

[2] Gross, G. and Doerr, H.W. (2003) Herpes Zoster Guidelines of the German Dermatological Society. Journal of Clinical Virology, 27, 308-309. https://doi.org/10.1016/S1386-6532(03)00125-2

[3] Gross, G., Schofer, H., Wassilew, S., Friese, K., Timm, A., Guthoff, R., Pau, H.W., Malin, J.P., Wutzler, P. and Doerr, H.W. (2003) Herpes Zoster Guideline of the German Dermatology Society (DDG). Journal of Clinical Virology, 26, 277-293. https://doi.org/10.1016/S1386-6532(03)00005-2

[4] Tyring, S., Belanger, R., Bezwoda, W., Ljungman, P., Boon, R. and Saltzman, R.L. (2001) A Randomized, Double-Blind Trial of Famciclovir versus Acyclovir for the 
Treatment of Localized Dermatomal Herpes Zoster in Immunocompromised Patients. Cancer Investigation, 19, 13-22. https://doi.org/10.1081/CNV-100000070

[5] Gopal, M.G., Sharath, S., Kumar, B.C., Nandini, A.S. and Namrata, C.M. (2013) A Comparative Study to Evaluate the Efficacy and Safety of Acyclovir and Famciclovir in the Management of Herpes Zoster. Journal of Clinical and Diagnostic Research, 7, 2904-2907.

[6] Gross, G. (2003) Zoster und Zosterschmerzen. Journal der Deutschen Dermatologischen Gesellschaft, 1, 398-407. https://doi.org/10.1046/j.1610-0387.2003.02005.x

[7] Wassilew, S. (1984) Management of Pain in Herpes Zoster. Seminars in Dermatology, 3, 116-119.

[8] Riopelle, J., Lopez-Anaya, A., Cork, R.C., Heitler, D., Eyrich, J., Dunston, A., Riopelle, A.J., Johnson, W., Ragan, A. and Naraghi, M. (1994) Treatment of the Cutaneous Pain of Acute Herpes Zoster with 9\% Lidocaine (Base) in Petrolatum/Paraffin Ointment. Journal of the American Academy of Dermatology, 30, 757-767. https://doi.org/10.1016/S0190-9622(08)81507-1

[9] Holcová, S. and Hladiková, M. (2015) Local Treatment-Accompanying Care for Herpes Zoster with an Active Substance-Containing Lotion (Propolis Special Extract GH 2002). The Journal of Pharmacology and Experimental Therapeutics, 24, 3-7.

[10] Walgrave, S.E., Warshaw, E.M. and Glesne, L.A. (2005) Allergic Contact Dermatitis from Propolis. Dermatitis, 16, 209-215. https://doi.org/10.1097/01206501-200512000-00004

[11] Holcová, S. and Hladiková, M. (2011) Efficacy and Tolerability of Propolis Special Extract GH 2002 as a Lip Balm against Herpes Labialis: A Randomized, Double-Blind Three-Arm Dose Finding Study. Health, 3, 49-56. https://doi.org/10.4236/health.2011.31010

[12] Holcová, S. and Hladiková, M. (2012) Inhibierung der Entwicklung von Lippenbläschen durch frühzeitige Anwendung eines pflegenden Lippenbalsams mit dem Wirkstoff Propolis Spezialextrakt GH 2002 im Vergleich mit Aciclovircreme 5\%. Kosmetische Medizin, 33, 100-104.

[13] Schnitzler, P., Neuner, A., Nolkemper, S., Zundel, C., Nowack, H. and Sensch, K.H. (2010) Antiviral Activity and Mode of Action of Propolis Extracts and Selected Compounds. Phytotherapy Research, 24, S20-S28. https://doi.org/10.1002/ptr.2868

[14] Nolkemper, S., Reichling, J., Sensch, K.H. and Schnitzler, P. (2010) Mechanism of Herpes Simplex virus Type 2 Suppression by Propolis Extracts. Phytomedicine, 17, 132-138. https://doi.org/10.1016/j.phymed.2009.07.006

[15] Astani, A., Zimmermann, S., Hassan, E., Reichling, J., Sensch, K.H. and Schnitzler, P. (2013) Antimicrobial Activity of Propolis Special Extract GH 2002 against Multidrug-Resistant Clinical Isolates. Pharmazie, 68, 695-701. 
Submit or recommend next manuscript to SCIRP and we will provide best service for you:

Accepting pre-submission inquiries through Email, Facebook, LinkedIn, Twitter, etc. A wide selection of journals (inclusive of 9 subjects, more than 200 journals)

Providing 24-hour high-quality service

User-friendly online submission system

Fair and swift peer-review system

Efficient typesetting and proofreading procedure

Display of the result of downloads and visits, as well as the number of cited articles Maximum dissemination of your research work

Submit your manuscript at: http://papersubmission.scirp.org/

Or contact health@scirp.org 